\title{
Generalised lymphangiomatosis: A diagnostic challenge
}

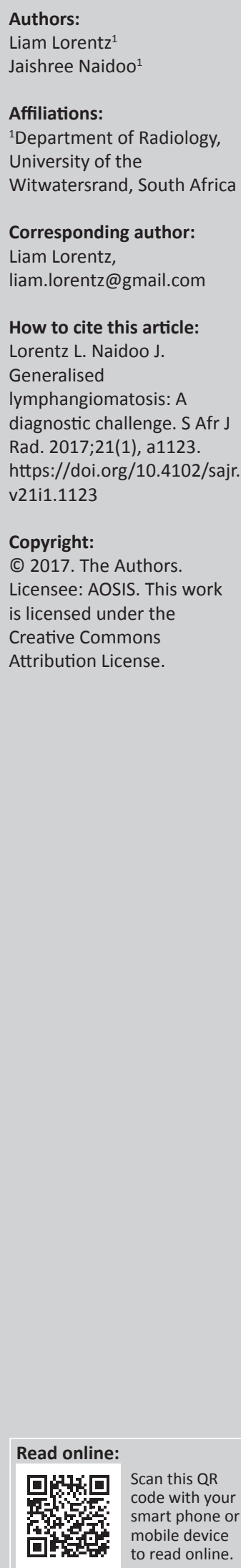

Background: Systemic, generalised lymphangiomatosis is a rare condition usually presenting in childhood or young adulthood. Characterised by proliferation of normal, mature lymphatic tissue, this benign disease may involve multiple organ systems. The brain is unaffected as it is devoid of lymphatic channels. The condition may ultimately result in severe, debilitating symptoms, and its aggressive nature may mimic malignancy. Because of its variable and unpredictable presentation, the evaluation of generalised lymphangiomatosis is a diagnostic challenge. The role of imaging is paramount in the diagnosis and establishing the extent of the disease.

Objectives: We present the case of a 3-year-old male with bleeding diatheses and hepatosplenomegaly clinically and large mediastinal mass on plain chest radiography. With a high index of suspicion for lymphoma, the child underwent multiple imaging modalities including chest and long bone radiography, abdominal ultrasound, contrast-enhanced chest and abdominal computed tomography (CT), with positron emission tomography (PET) and chest and abdominal magnetic resonance imaging (MRI).

Method: Imaging demonstrated a cystic, mediastinal mass encompassing the major vessels, pericardium and trachea. Similar masses were also located within the abdomen encasing the aorta. CT demonstrated splenomegaly with multiple low attenuation foci. There was no focal increase in 19-FDG uptake on PET/CT imaging. MRI confirmed multiple areas of bone marrow involvement.

Results: Histological evaluation of the bone marrow trephine was inconclusive. Biopsy of the mediastinal mass was histopathologically described as cavernous lymphangioma.

Conclusion: This case illustrates the obscure presentation and variable course of generalised lymphangiomatosis. Whole body short tau inversion recovery (STIR) MRI provides an indication of the extent of the disease including bone marrow infiltration. The prime clinical dilemma is exclusion of malignancy. Multi-disciplinary discussion with multi-modality imaging is required to diagnose and manage the condition.

Note: A selection of conference abstracts: RSSA/SASPI Paediatric Imaging Congress, 03-06 November 2016, Spier Estate, Stellenbosch, South Africa. Faculty collaborators: Professor Kassa Darge (Body Imaging, University of Pennsylvania, Philadelphia, USA), Professor Edward Lee (Thoracic Imaging, Harvard University, USA), Professor Beverley Newman (Cardiac Imaging, Stanford University, California, USA), Professor Kimberly Applegate (Image Gently and Body Imaging, Emory University, Atlanta, USA) and Professor Savvas Andronikou (Thoracic Imaging, University of Bristol, UK) supported by South African Paediatric Radiologists, co-ordinated by Dr. Jaishree Naidoo, President of the African Society of Paediatric Imaging and Head of Division of Paediatric Radiology, Charlotte Maxeke Johannesburg Academic Hospital. 